\title{
Msx1 et son influence sur la croissance cranio-faciale
}

\author{
Brigitte Vi-Fane $^{a *}$, Isabelle Fernandes ${ }^{b}$, Jean-Luc Davideau ${ }^{c}$ \\ a Université Denis Diderot (Paris VII), Faculté de Chirurgie Dentaire, 5 rue Garancière, 75006 Paris, France \\ b UMRS 872 - Équipe 5 - Biologie Orofaciale et Pathologie, 15-21 rue de l'École de Médecine, 75270 Paris Cedex 06, France \\ c Université Louis Pasteur, UFR d'Odontologie, 1 place Hôpital, 67000 Strasbourg, France
}

RÉSUMÉ - Le développement de la sphère cranio-faciale implique de nombreux gènes qui interviennent de façon complexe et interdépendante. Parmi ces gènes, l'homéogène Msx1 est un facteur de transcription, qui est exprimé, des stades précoces du développement jusqu'à l'âge adulte selon des schémas spatio-temporels spécifiques. Son invalidation, chez des souris transgéniques qui présentent alors diverses anomalies cranio-faciales, a mis en évidence son impact fonctionnel, tout comme l'identification de certaines mutations chez l'humain dont la caractéristique commune est l'agénésie dentaire.

MOTS CLÉS - Msx1 / Souris transgéniques / Mutations / Anomalies cranio-faciales / Agénésie

\begin{abstract}
Many genes that interact in a complex and interdependent manner participate in the development of the craniofacial complex. One of them, the Msx1 homeobox gene, a transcription factor, is expressed from early developmental stages to adulthood in accordance with specific spatio-temporal patterns. When it is suppressed, transgenic mice exhibit craniofacial abnormalities that demonstrate what is its function in normal growth, just as it has been shown that certain Msx1 mutations in humans are commonly associated with tooth agenesis.
\end{abstract}

KEYWORDS - Msx1 / Transgenic mouse models / Mutations / Craniofacial abnormalities / Agenesis

\section{Introduction}

La croissance cranio-faciale est un processus dynamique qui implique la coopération de nombreux types cellulaires tels que les cellules de l'épithélium oral et les cellules des crêtes neurales céphaliques qui se caractérisent par leur capacité de migration, de la surface dorsale du tube neural vers les arcs branchiaux, et de formation des dérivés squelettiques ectomésenchymateux. Elle requiert une régulation précise des mouvements, de la détermination et de la différenciation des cellules constituant les tissus cranio-faciaux.

Elle est contrôlée par des facteurs spécifiques, facteurs de croissance, facteurs de transcription et protéines matricielles, qui interviennent à toutes les étapes de ce processus du développement. Ces facteurs peuvent être codés soit par des gènes initiaux qui contrôlent les patrons du développement, la différenciation cellulaire et la morphogenèse initiale, soit par des gènes terminaux qui s'expriment pendant les étapes de formation et de minéralisation

\footnotetext{
*Auteur pour correspondance : brigitte.vi-fane@wanadoo.fr
}

des tissus squelettiques cranio-faciaux, soit par des gènes dits de croissance qui contrôlent la prolifération cellulaire, la morphogenèse terminale et les phénomènes de croissance, soit enfin par des gènes plus ubiquitaires et généralistes (cette classification étant simplement didactique). Toute mutation de ces gènes a un impact sur le développement et la croissance cranio-faciale et engendre une anomalie plus ou moins importante, isolée ou syndromique, en fonction de leur degré de spécificité et de leur période d'intervention.

L'exemple type des interactions cellulaires et de leurs régulations est illustré au cours de la morphogenèse dentaire [10, 44] (Fig. 1). Les dents des mammifères, comme de nombreux autres organes, se développent grâce à une série d'interactions épithélio-mésenchymateuses. Ces interactions inductives, réciproques et séquentielles, entraînent les changements transcriptionnels nécessaires à la progression du développement dentaire. Cela a été montré notamment par des expériences de dissociations/réassociations in vitro et in vivo $[24,28,45]$. 


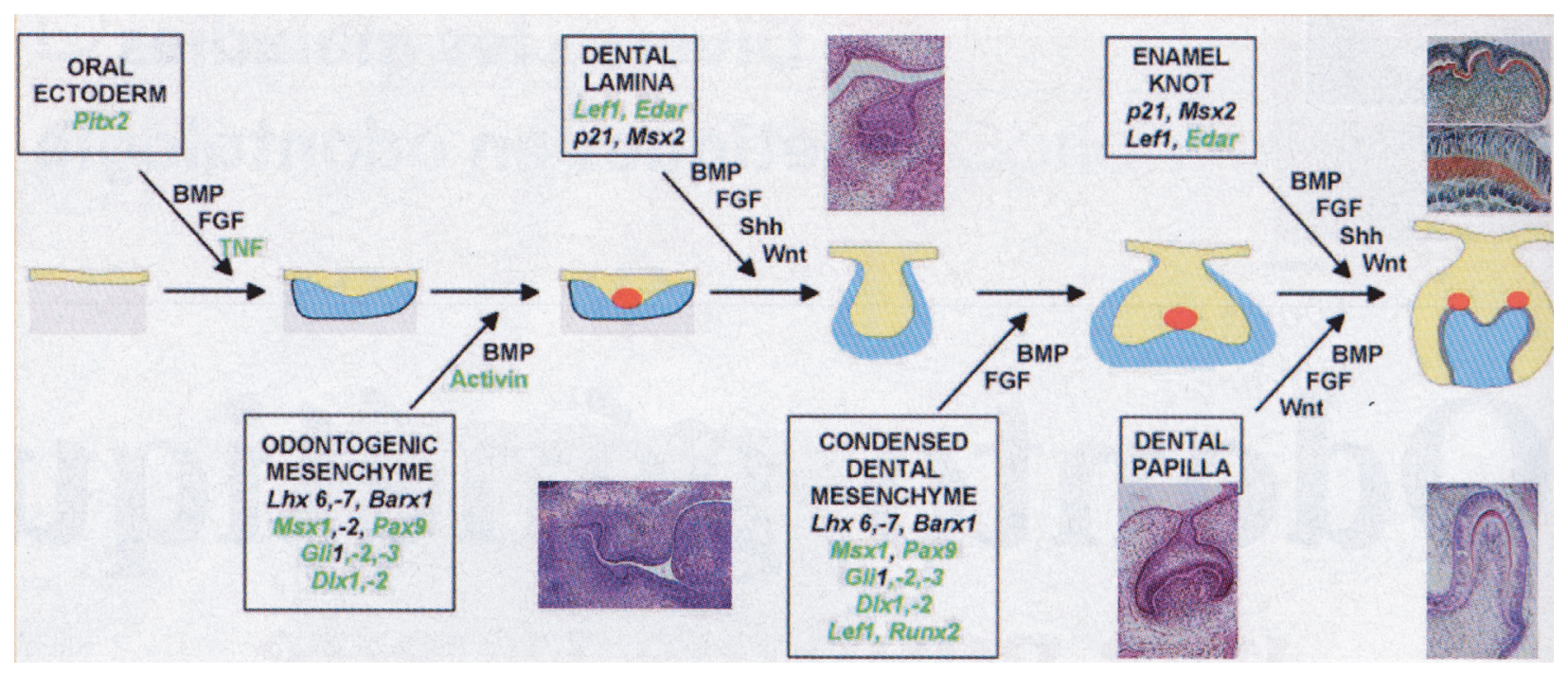

Figure 1

Représentation schématique des voies de signalisation régulant l'odontogenèse. (D'après Thesleff et Mikkola [44], droits réservés.) Les événements morphogénétiques de l'odontogenèse sont :

- l'initiation, caractérisée par l'apparition de la lame dentaire;

- la morphogenèse au cours de laquelle les germes s'individualisent suivant les étapes de bourgeon, de cupule jeune puis âgée et de cloche dentaire;

- la cytodifférenciation des améloblastes, des odontoblastes et des cémentoblastes. S'en suivent l'apposition et la minéralisation coronaire puis radiculaire, l'éruption s'achevant par la formation terminale de la racine.

Ces étapes morphologiques sont la résultante d'échanges organisés de signaux entre les cellules de l'épithélium oral et celles de l'ectomésenchyme. Les signaux ou ligands sont parfois réutilisés au cours de différentes étapes et souvent dans différentes directions.

$\mathrm{Au}$ cours du développement embryonnaire, chaque région du complexe cranio-facial est formée sous le contrôle de combinaisons d'expression de gènes à homéoboîte divergents ou non-hox selon des schémas spatio-temporels spécifiques [6]. Certains de ces gènes semblent jouer un rôle au cours de la croissance postnatale.

Parmi ces gènes, la famille des gènes Msx ( $\underline{\text { Muscle }}$ segment homeobox gene), homologues du gène Msh de la drosophile, joue un rôle clé comme le montrent l'étude des souris transgéniques et l'identification des mutations de ces gènes responsables de certaines malformations cranio-faciales chez l'homme. Chez les deux espèces, les phénotypes observés sont similaires, justifiant l'utilisation de la souris comme modèle d'étude pour l'approche expérimentale des dysmorphoses humaines. Ces gènes Msx constituent une petite famille codant des facteurs de transcription contenant un homéodomaine et sont dispersés dans le génome. Ils s'expriment notamment dans les cellules des crêtes neurales et au cours des interactions épithélio-mésenchymateuses déterminant le développement cranio-facial et celui des bourgeons de membre. Ce sont des acteurs de premier rôle lors de la mise en place des tissus dentaires et osseux $[25,41,46,47,58,59]$.

Chez l'homme, deux gènes (Msx1 et Msx2) ont été identifiés et localisés respectivement sur les chromosomes 4 et 5 , alors que chez la souris, trois gènes (Msx1, Msx2 et Msx3) ont été isolés [12, 39, 57], situé sur les chromosomes 5,13 et 7 . Lhoméogène Msxl qui nous intéresse a été appelé Hox-7 en raison de son homéoboîte similaire à celui découvert initialement dans les homéogènes de type Hox $[26,39,52]$; comme il n'appartenait pas au complexe Hox, ce terme a été abandonné. Le gène Msxl se singularise par un mode original d'expression tissulaire et de régulation via un ARN antisens endogène [3]. Son rôle pendant le développement anténatal est illustré de façon exemplaire au niveau de la formation des dents et du squelette craniofacial [6].

\section{Msxl}

\subsection{Généralités}

Le gène Msxl murin est composé de deux exons, de 590 et 1214 paires de bases (pb) séparés par un intron de $2107 \mathrm{pb}$ avec l'homéoboîte située dans la 


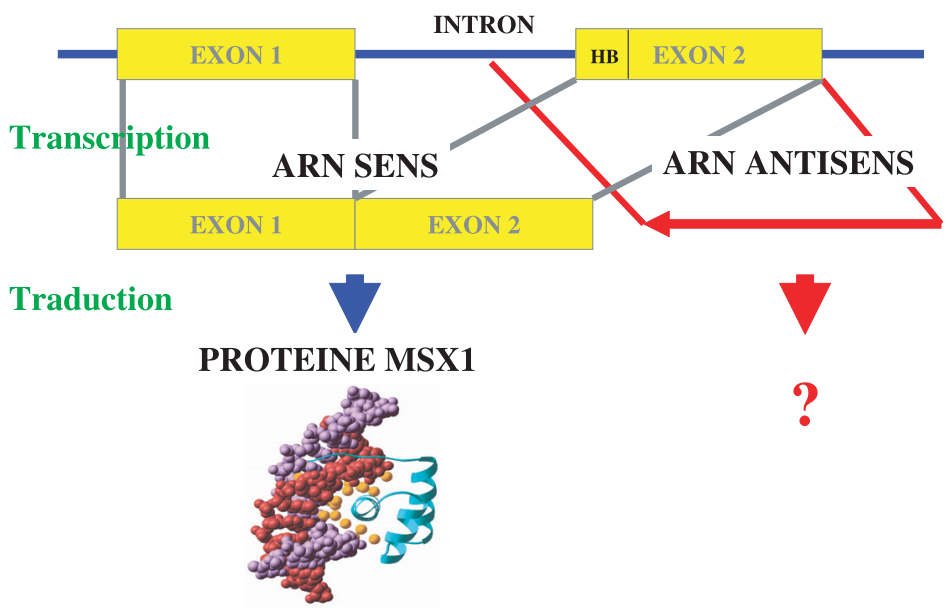

Figure 2

Schéma du gène Msx1. Le transcrit sens résulte de la transcription du brin $A D N$ codant du gène après excision-épissage du transcrit d'intron. Le transcrit antisens résulte de la transcription du brin ADN non codant de l'exon 2 et d'une partie de l'intron. La protéine MSX1 présente un homéodomaine de 60 acides aminés formant trois hélices ayant la structure canonique hélice tour hélice (bleu). La troisième hélice ou l'hélice de reconnaissance de l'ADN s'adapte au grand sillon de l'ADN parallèlement au plan des bases de l'ADN (rouge et parme). Cette hélice est responsable de la majorité des interactions protéine-ADN [14] (HB : homéoboîte). première partie du second exon (Fig. 2). Les sites d'épissage et de polyadénylation sont conformes à ceux de la plupart des gènes de vertébrés. Le site d'initiation de la transcription est situé à 256 pb en amont du premier AUG.

Il présente un site de fixation de la protéine MSX1 au niveau de son promoteur proximal, permettant d'envisager une autorégulation par la protéine MSX1 même, et/ou une régulation croisée par la deuxième homéoprotéine MSX2 très proche dans ses motifs de liaison à l'ADN $[13,22]$. En se fixant sur ce site, MSX1 serait capable de réprimer la transcription de son propre gène en formant un complexe protéique avec d'autres protéines (Spl, TBP ou encore CBP/p300) [43].

Deux transcrits sont décrits pour Msxl : un ARN sens de $1804 \mathrm{pb}$ et un ARN antisens de $2184 \mathrm{pb}$. L'ADNc de Msxl antisens est complémentaire de la région s'étendant depuis la région 3' de l'exon 2 jusqu'au milieu de l'intron de la séquence génomique de Msxl [3]. Cet ARN antisens est un des rares cas de long antisens qui possèdent une si longue complémentarité avec leur transcrit sens $[21,60]$. Contrairement au transcrit sens, ce transcrit antisens ne semble pas traduit en protéine. Il possède cependant une petite phase de lecture ouverte qui pourrait théoriquement être traduite en une protéine. Cependant, aucune séquence répertoriée dans les banques de données n'a été rapportée jusqu'alors. Des études in vitro ont montré que cet ARN antisens était capable d'inhiber l'expression du transcrit sens en protéine. Le mécanisme impliqué se situerait au niveau post-transcriptionnel et se ferait par dégradation des ARN sens (Petit, et al., article en préparation).
La structure du gène humain Msxl est proche de celle du gène murin avec ses deux exons séparés par un intron de 1,6 kb. Les régions transcrites non codantes des gènes humain et murin sont étonnamment conservées au niveau des régions 5', tout comme les régions 3'.

La protéine MSX1 compte 297 acides aminés et a un poids moléculaire de $36451 \mathrm{Da}$ chez la souris [61]. Comme pour de nombreuses protéines à homéodomaine, un site consensus de liaison de l'ADN, construit autour d'un cœur TAAT, a été défini pour Msxl [4]. La protéine MSX1 est un répresseur de la transcription, et ceci dépendamment ou indépendamment de son activité de liaison à l'ADN. En effet, il a été montré que la fixation de MSXl sur sa séquence de reconnaissance n'est pas nécessaire à sa fonction de répression de la transcription [4].

\subsection{Fonctions de Msxl}

Les fonctions sont démontrées ou suggérées pour la protéine MSX1. Elle semble intervenir au cours des différents phénomènes cellulaires qui gouvernent le développement (induction, maintien d'une certaine multipotence, prolifération et différenciation cellulaires, apoptose).

- MSXI est associée à la détermination de champs morphogénétiques notamment dans l'œil [29] ou au niveau dentaire, en particulier le champ présomptif incisif [50]. En effet, chez les souris pour lesquelles le gène Msxl est inactivé, aucun développement dentaire incisif n'est initié [40].

- Au cours de l'organogenèse, MSXI est fortement impliquée dans les interactions épithéliomésenchymateuses, essentielles au développement 
et à la croissance de certains organes, comme l'œil [29], les follicules pileux, les dents [2, 40].

- L'expression de MSX1 est généralement observée dans des territoires en pleine prolifération, ou qui gardent une certaine plasticité. Dès que la différenciation cellulaire s'amorce, l'expression de MSX1 s'éteint. Ainsi, MSX1 contrôle la prolifération et le maintien d'un état multipotent des cellules progénitrices mésenchymateuses et épithéliales qui peuvent devenir des cellules soit musculaires, soit cartilagineuses, soit osseuses ou encore adipeuses ou dentaires [15].

- MSX1 joue un rôle clé dans la plasticité cellulaire en assurant une multipotentialité cellulaire au cours du développement et de la croissance et chez l'adulte. Il peut, notamment en expérimentation, induire la dédifférenciation des myotubes et leur transdifférenciation en ostéoblaste dans des conditions de culture permissives [33].

- Certaines données semblent impliquer MSX1 dans la mort cellulaire programmée - il aurait un rôle de facteur pro-apoptotique $[8,49]$ - et dans les phénomènes de régénération [38].

\subsection{Expression de Msxl au cours du développement anténatal et postnatal}

La majorité des études sur les schémas d'expression de Msxl concernent exclusivement le transcrit sens de Msxl. Mais l'utilisation de souris transgéniques présentant une invalidation du gène, soit par « knock-out» (KO), soit par « knock-in » (KI), a permis d'aborder la non-expression (KO) ou l'expression d'une protéine chimère MSX1 non fonctionnelle (KI).

L'expression de Msxl est globalement indépendante de la lignée cellulaire. Son expression semble plus répondre à la situation spatiale du tissu et des interactions que celui-ci subit au sein d'un groupe de cellules hétérogènes. Msxl est principalement présent dans des zones où se produisent des interactions épithélio-mésenchymateuses. Msxl est détecté aussi bien dans l'épithélium que dans le mésenchyme; cependant, dans la majorité des cas, il ne l'est que dans l'un ou l'autre de ces tissus qui constitue l'organe en formation [27]. Les limites d'expression semblent davantage respecter une logique de gradient, par exemple proximo-distal dans le mésenchyme mandibulaire et maxillaire $[34,41]$ qu'une logique de différenciation cellulaire.
Msxl est exprimé précocement dès le stade de la gastrulation, dans le mésoderme et dans l'ectoderme de la ligne primitive [42]. À ce stade, les cellules extra-embryonnaires et maternelles telles que l'utérus, principalement l'épithélium utérin et les glandes tubulaires utérines ou le cordon ombilical expriment aussi Msx1 [8]. À partir des stades de développement précoce de post-gastrulation, l'expression de Msxl se restreint au mésenchyme sous la gouttière neurale, à l'ectoderme latéral et au neuroépithélium [39]. Cette expression se concentre progressivement dans la région dorsale du tube neural et dans le mésenchyme sous-jacent d'où émergent les cellules des crêtes neurales céphaliques $[12,39]$. Lors de leur migration, les cellules des crêtes neurales continuent d'exprimer Msxl ; cette expression se poursuit après leur migration et leur colonisation des arcs branchiaux, notamment dans les bourgeons cranio-faciaux $[13,26]$. Dans le futur système nerveux central, l'expression Msxl se limite à des zones très précises telles que l'ébauche du thalamus, la surface des troisième et quatrième ventricules cérébraux en formation [26]. Par ailleurs, Msxl est exprimé au niveau des méninges du cerveau en développement ainsi que dans les zones où les futurs os du crâne se formeront [27]. Au cours du développement précoce du premier arc, Msxl est également exprimé dans l'épithélium et le mésenchyme sous-jacent des bourgeons nasaux latéraux et médians $[12,26,27]$, ce qui n'est pas sans rappeler ce qui est observé dans le patron de mise en place de la zone de progression du membre $[26,27]$ et il existe un gradient disto-proximal dans le mésenchyme mandibulaire et maxillaire [41].

Au cours de l'organogenèse et des stades tardifs du développement anténatal, l'expression de Msxl se poursuit selon des schémas spatiaux particuliers correspondant à des territoires morphogénétiques (odontogène, crânien...) (Vi-Fane, et al., article en préparation).

Msxl est retrouvé au niveau des sites anatomiques cranio-faciaux où les interactions cellulaires ont une importance fonctionnelle. Msxl est exprimé dans la zone de mésenchyme qui entoure le méat auditif en formation, dans le mésenchyme dérivé des $2^{\mathrm{e}}$ et $3^{\mathrm{e}}$ arcs branchiaux qui formeront plus tard les composants de l'oreille moyenne et l'apophyse styloïde [26]. À proximité du prosencéphale, il est exprimé par les cellules mésenchymateuses dans des territoires préfigurant les ébauches des os du crâne 


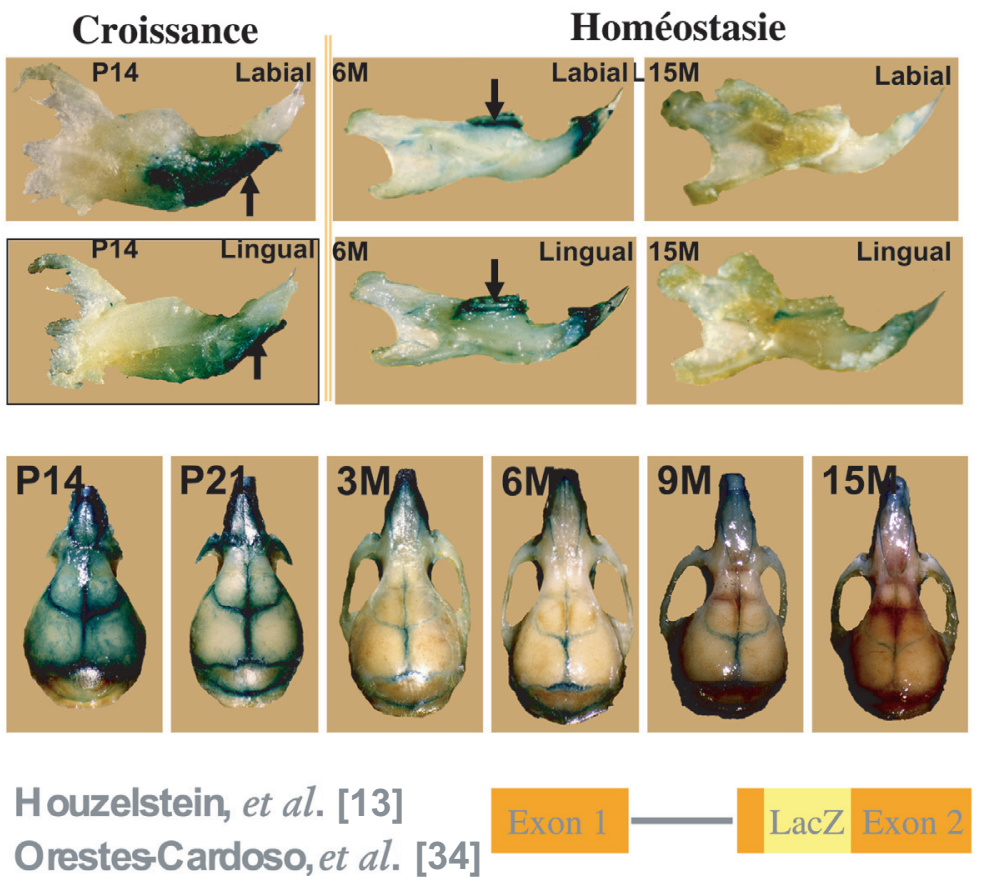

Figure 3

Expression postnatale de MSX1 (de 14 jours à 15 mois). L'expression de la protéine MSX1 est étudiée sur des souris transgéniques hétérozygotes Msx1/nLacZ. Durant cette période, MSX1 s'exprime selon un double gradient disto-proximal et aboral-oral au niveau du bord inférieur du corps de la mandibule. Mais on note une expression temporelle variable de la protéine au niveau de l'os alvéolaire. L'expression de MSX1 est continue au niveau des sutures et dans la partie antérieure du museau (os nasaux et cartilages); mais elle tend à diminuer avec l'âge. (frontal, temporal, pariétal et occipital) et dans les régions distales des bourgeons maxillaires et mandibulaires et plus tard dans les centres d'ossification des os des mâchoires [27]. Des études ont montré que Msxl est essentiel à la formation du squelette cranio-facial primaire constitué d'os fibrillaire et de cartilage [13, 19, 34, 40]. Au niveau mandibulaire, le schéma d'expression de Msxl décrit un double gradient disto-proximal et aboral-oral au niveau du bord inférieur de la mandibule. Par ailleurs, il a été rapporté une expression faible et diffuse de Msxl dans le palais en formation prouvant que Msx1 pourrait avoir un rôle direct dans son développement $[1,26,51]$. Cette expression de Msxl dans le mésenchyme palatin est cantonnée à la partie antérieure des bourgeons du palais $[1,62]$ et tend à diminuer progressivement [32].

L'expression de Msxl se poursuit après la naissance, au cours de la croissance postnatale et jusqu'à l'âge adulte. Msxl reste exprimé au niveau de certaines zones de croissance osseuse comme les sutures crâniennes et le demeure tout au long de la vie $[34,35]$. Par ailleurs, les zones d'expression au niveau facial varient au cours des différentes étapes de la croissance. Par exemple, à la mandibule, le schéma d'expression de Msxl montre un double gradient aboral-oral et disto-proximal au niveau du bord inférieur du corps mandibulaire lors des premières semaines postnatales; puis vers le $3^{\text {e }}$ mois, Msxl est retrouvé essentiellement au niveau de l'os alvéolaire. Au niveau des sutures craniofaciales, l'expression de Msxl persiste tout au long de la vie avec cependant une tendance à diminuer avec l'âge [34, 35] (Fig. 3). Ainsi l'expression de Msx1, chez l'adulte, est observée dans des sites où Msxl est impliqué au cours du développement squelettique initial [34] ; cependant certains os en formation (frontal, nasal, pariétal, lacrymaux) expriment Msxl avec une organisation anatomique plus claire que dans les stades anténataux (Vi-Fane, et al., article en préparation).

Par ailleurs, de la naissance à 15 mois postnatal, dans le cerveau mature de la souris, Msxl est exprimé dans les organes localisés autour du $3^{\mathrm{e}}$ ventricule (circumventricular) comme l'organe sous-commissural, qui présente d'ailleurs un développement anormal chez les souris Msxl «knockout » [36], et dans le plexus choroïde ainsi que dans la partie dorsale du $3^{e}$ ventricule. La présence de Msxl dans ces régions serait associée à l'activité sécrétoire de ces organes ainsi qu'à la prolifération et à la différenciation des cellules gliales notamment dans l'hippocampe, faisant de Msxl un acteur de l'homéostasie cérébrale [37]. De façon intéressante, d'autres tissus adultes, comme l'utérus et les glandes mammaires, à renouvellement tissulaire sous dépendance hormonale cyclique expriment aussi l'homéoprotéine Msxl. 


\section{0 jour}
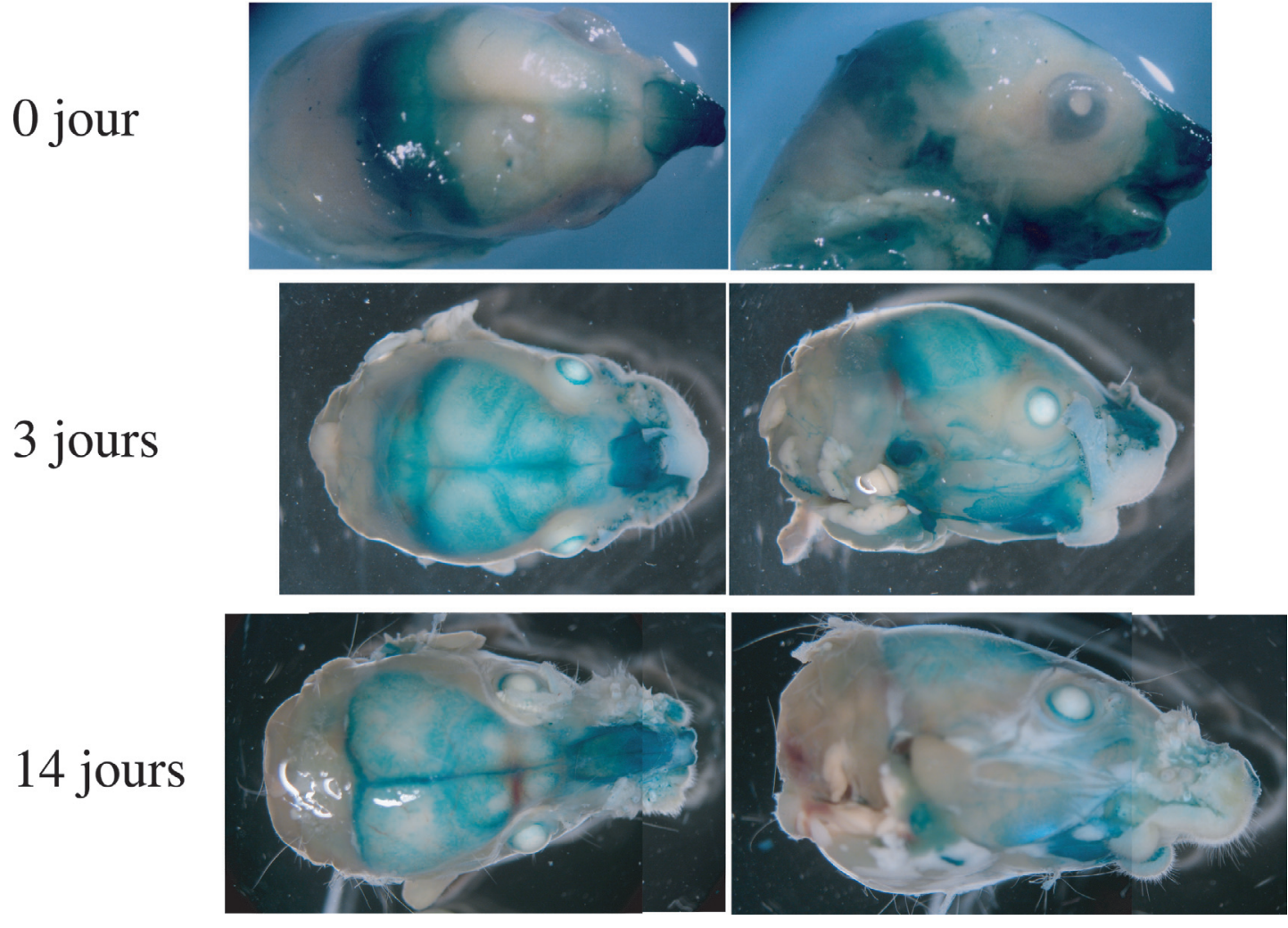

Figure 4

Expression postnatale de MSX1 (naissance à 14 jours). La protéine MSX1 est détectée au niveau des sites de croissance squelettique, tant au niveau du crâne dont la croissance se fait de façon radiée qu'au niveau facial (os nasaux, mandibule, maxillaire).

Au cours de la croissance, l'expression de Msxl est tissu-spécifique et fonction du site, notamment au niveau des sites de modelage actif du squelette. Msxl est ainsi exprimé dans le mésenchyme associé aux régions chondroprogénitrices (cartilage de Meckel, par exemple), ostéoprogénitrices d'os de membrane (mandibule, maxillaires, crâne) et aux sutures (massif cranio-facial) (Fig. 4). Au niveau cellulaire, les cellules hétérogènes du squelette expriment Msxl, certains chondrocytes lors de l'ossification endochondrale, tous les préostéoblastes, certains ostéoblastes et ostéocytes lors de l'ossification membraneuse, tous les chondroclastes des cartilages de croissance et certains ostéoclastes des os en croissance $[34,56]$. La croissance achevée, l'expression de Msxl se maintient pendant les étapes d'homéostasie, expression qui correspondrait à des sous-populations de cellules souches ou cellules progénitrices locales.

Lannulation fonctionnelle du gène Msxl, chez la souris, permet de déterminer son impact fonctionnel et sa régulation au cours du développement et de la croissance. Il existe deux modèles de souris transgéniques d'invalidation du gène Msxl. Lun correspond à l'insertion du gène de résistance à la néomycine [40] donnant la souris « knock-out », l'autre à l'insertion du gène rapporteur nLacZ [13] donnant une souris «knock-in»; les deux insertions sont réalisées à l'intérieur de l'homéoboîte par recombinaison homologue. Dans le deuxième modèle, d'une part, le gène est inactivé par destruction d'une région considérée comme indispensable à sa fonction et, d'autre part, il y a création d'une protéine chimère, de fusion repérable par son activité bétagalactosidase et facilement détectable. Dans les deux cas, les mutants homozygotes Msxl -/- meurent à la naissance.

Ces animaux homozygotes Msxl -/- présentent des défauts dans la région cranio-faciale, en particulier une fente totale du palais secondaire, des défauts de l'oreille moyenne et des os nasaux, une série de dysmorphies au niveau du crâne et des maxillaires, et des agénésies par blocage du développement dentaire $[13,40]$. Leur tête est plus petite 
et plus ronde; ces caractéristiques sont frappantes au niveau du museau. Les os nasaux sont plus rectangulaires et plus courts. Les anomalies crâniennes concernent principalement le frontal et les pariétaux et se manifestent par un élargissement de la fontanelle antérieure et de la suture métopique. Au niveau de l'oreille moyenne, seul le marteau de la chaîne des osselets est affecté; il présente une taille réduite et son apophyse courte est agénésique. Le prémaxillaire est absent. La fente palatine est due davantage à un développement insuffisant des processus palatins issus des os maxillaires et du palatin que d'une anomalie de redressement de ces derniers. Leur absence permet une visualisation directe du vomer et du sphénoïde. La mandibule perd sa forme générale en U et dessine un $\mathrm{V}$; la convexité de la base mandibulaire disparaît, le condyle a un aspect plus allongé et plus fin $[34,40]$. Par ailleurs, on note une absence d'os alvéolaire tant au niveau des molaires qu'au niveau des incisives. Le développement des molaires s'arrête au stade de bourgeon, le mésenchyme semblant incapable d'induire la différenciation de l'épithélium oral. Les incisives sont, elles, agénésiques $[34,40]$. Toutes ces anomalies se manifestent au niveau des zones d'expression de Msxl. Contre toute attente, il n'y a aucun défaut au niveau des membres qui sont pourtant le site majeur d'expression de Msxl et le lieu de la découverte de son rôle dans les interactions épithéliomésenchymateuses.

La comparaison du schéma d'expression de la protéine Msxl avec le phénotype de la souris mutant nul Msx1-/- [34,35] souligne l'influence quantitative de Msxl dans le squelette cranio-facial.

Chez l'homme, plusieurs (12 au moins) mutations et délétions de Msxl ont été identifiées [5, $9,11,18,20,23,30,31,48,53,54]$. Leur caractéristique phénotypique commune est l'agénésie dentaire syndromique ou non syndromique; des anomalies cranio-faciales y sont parfois associées. Plus de 60 syndromes s'accompagnent d'anomalies dentaires. Cette donnée suggère que les mécanismes moléculaires impliqués dans le développement et la croissance de la dent sont similaires à ceux d'autres organes.

L'expressivité et la pénétrance de ces mutations du gène Msxl ont été associées à l'haploinsuffisance $[16,17,31,53]$. Une mutation faux-sens dans l'exon 2 due à la substitution d'une arginine par une proline entraine l'agénésie sélective des prémolaires et des molaires $[16,54]$. Une mutation nonsens de Msxl a été associée à des agénésies dentaires maxillaires et mandibulaires, atteignant indifféremment toutes les dents et liées à des combinaisons variables de fentes palatines isolées ou labio-palatines non-syndromiques [53]. Une autre a été associée au syndrome de Witkop, aussi nommé TNS (Tooth Nails Syndrome), syndrome rare transmis selon un mode autosomique dominant et appartenant à la famille des dysplasies ectodermiques [18]. Enfin, la délétion du gène Msxl est associée au syndrome de WolfHirschhorn, résultant de la perte du bras court du chromosome 4 [31]. Par ailleurs, un important travail de caractérisation des mutations et des polymorphismes de ce gène a été entrepris par l'équipe de Murray [17].

\subsection{Transcrit antisens de Msx 1}

Msxl présente une caractéristique originale par sa transcription bidirectionnelle. Un ARN antisens non codant est transcrit à partir du même locus que l'ARN sens qui, lui, est codant. Cet ARN antisens endogène de Msxl a été identifié initialement dans les cellules dentaires et osseuses différenciées, chez les rongeurs et montré exister chez l'humain [3]. Sa structure particulière implique la possibilité de formation de duplex entre ARN sens et antisens, qui pourraient influencer les mécanismes de régulation de la synthèse du facteur actif, l'homéoprotéine MSXI. In vitro, ce transcrit antisens inhibe l'expression de la protéine MSX1 [3]. Lanalyse in vivo de l'expression des produits de Msxl a mis en évidence l'impact de l'ARN antisens sur l'expression de la protéine MSX1 pendant la formation osseuse craniofaciale et sa croissance.

Chez la souris, le transcrit antisens de Msxl a été détecté au niveau cranio-facial et au cours du développement dentaire [3], de E12.5 à quelques jours après la naissance (Fig. 5). Lhybridation in situ des deux transcrits de Msxl montre des schémas d'expression spatio-temporelle spécifiques [55] suivant des localisations différentes et une co-expression lors de la différenciation cellulaire. Le transcrit antisens est présent dans le mésenchyme et l'épithélium dentaires alors que le transcrit sens n'est localisé que dans le mésenchyme dentaire [3]. Dans les os maxillaires et mandibulaire, l'antisens est détecté dans les cellules osseuses très différenciées que sont les 

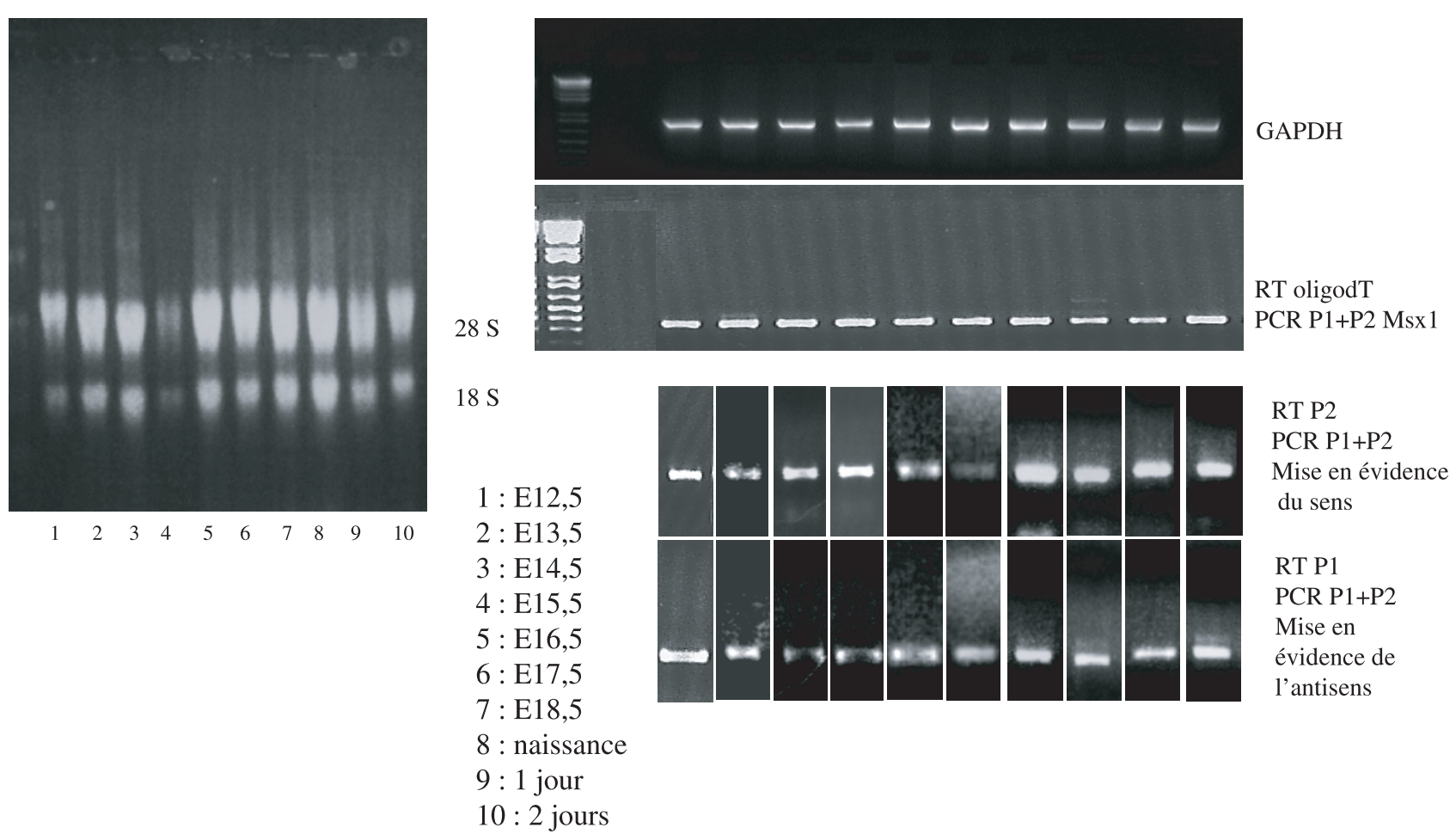

Figure 5

Expression des transcrits sens et antisens de Msx1. Gel des ARNs totaux et des produits de RT-PCR. Les ARNs totaux sont extraits à partir de prélèvements broyés puis transcrits en ADNc à partir d'amorces spécifiques sens et antisens et non spécifiques. Ces produits sont ensuite amplifiés par PCR (Polymerase Chain Reaction) à partir de combinaisons d'amorces spécifiques, puis analysés sur gel. Les deux transcrits sens et antisens de Msx1 sont co-exprimés chez la souris, du stade E12.5 à deux jours après la naissance.

ostéocytes inclus dans la matrice osseuse et les ostéoblastes, alors que le sens et la protéine sont détectés dans les cellules osseuses progénitrices et en cours de différenciation. Cet ARN antisens a également été détecté dans les follicules pileux, dans les bourgeons de membres [7] et dans de nombreux organes de souris adulte tels que les poumons, le foie, ou encore le cœur.

Ce transcrit antisens est par ailleurs impliqué dans la régulation des interactions existantes entre les membres des familles de protéines MSX et DLX; la surexpression de Dlx5 est capable d'inhiber l'expression du transcrit antisens [3].

Msxl possède un autre système de régulation, par son transcrit antisens naturel, comme d'autres homéogènes tels deux membres de la famille des Dlx, famille très proche de la famille Msx, Dlx1 et Dlx6 ou encore trois (voire quatre) membres de la famille des Hox (Hoxb-3, Hoxd-3 et Hoxal1). Ces couples S/AS sont souvent impliqués dans les « activités de régulation de la transcription ». Ainsi, la balance sens et antisens jouerait un rôle dans le contrôle de la croissance cranio-faciale.

\section{Conclusions}

L'ensemble des données expérimentales et les différentes études cliniques portant sur les mutations humaines de Msxl montrent l'importance de l'homéogène Msxl au cours du développement craniofacial. Son expression prolongée jusqu'à l'âge adulte laisse deviner une influence au cours de la croissance et de l'homéostasie; par ses trois produits (protéine, transcrits sens et antisens) qui constituent une unité fonctionnelle, Msxl est impliquée dans des voies de régulation plus fine. Par ailleurs, les phénotypes associés aux mutations de Msxl sont divers et variables, allant de la simple agénésie dentaire à des syndromes plus importants, notifiant que d'autres facteurs régulent la pénétrance des anomalies observées, notamment les facteurs environnementaux et le polymorphisme génétique et soulignant la complexité de la génétique du développement et la croissance de la sphère cranio-faciale. 


\section{Bibliographie}

[1] Alappat S, Zhang ZY, Chen YP. Msx homeobox gene family and craniofacial development. Cell Res 2003;13:429-442.

[2] Bendall AJ, Abate-Shen C. Roles for Msx and Dlx homeoproteins in vertebrate development. Gene 2000;247:7-31.

[3] Blin-Wakkach C, Lézot F, Ghoul-Mazgar S, Hotton D, Monteiro S, Teillaud C, et al. Endogenous Msxl antisense transcript: In vivo and in vitro evidences, structure, and potential involvement in skeleton development in mammals. Proc Natl Acad Sci U S A 2001;98(13):7336-7341.

[4] Catron KM, Zhang H, Marshall SC, Inostroza JA, Wilson JM, Abate C. Transcriptional repression by Msx-1 does not require homeodomain DNA-binding sites. Mol Cell Biol $1995 ; 15: 861-871$.

[5] Chishti MS, Muhammad D, Haider M, Ahmad W. A novel missense mutation in MSX1 underlies autosomal recessive oligodontia with associated dental anomalies in Pakistani families. J Hum Genet 2006;51(10):872-878.

[6] Cobourne MT, Sharpe PT. Tooth and jaw: molecular mechanisms of patterning in the first branchial arch. Arch Oral Biol 2003;48(1):1-14.

[7] Coudert A. Étude de l'expression et de la régulation du transcrit antisens du gène Msxl au cours du développement embryonnaire : approches expérimentales dans l'odontogenèse. Thèse Doct État. Paris: Univ P. et M. Curie, 2005.

[8] Davidson D. The function and evolution of Msx genes: pointers and paradoxes. Trends Genet 1995;11:405-411.

[9] De Muynck S, Schollen E, Matthijs G, Verdonck A, Devriendt K, Carels C. A novel MSX1 mutation in hypodontia. Am J Med Genet A 2004;128(4):401-403.

[10] Francis-West FH, Robson L, Evans DJR. Craniofacial development: The tissue and molecular interactions that control development of the head. Adv Anat Embryol Cell Biol 2003;169:III-VI,1-138.

[11] Frazier-Bowers SA, Guo DC, Cavender A, Xue L, Evans B, King T, et al. A novel mutation in human PAX9 causes molar oligodontia. J Dent Res 2002;81:1296-1333.

[12] Hill RE, Jones PF, Rees AR, Sime CM, Justice MJ, Copeland NG, et al. A new family of mouse homeoboxcontaining genes: molecular structure, chromosomal location, and developmental expression of Hox-7.1. Genes Dev 1989;3:26-37.

[13] Houzelstein D, Cohen A, Buckingham ME, Robert B. Insertional mutation of the mouse Msxl homeobox gene by an nlacZ reporter gene. Mech Dev 1997;65:123-133.

[14] Hovde S, Abate-Shen C, Geiger JH. Crystal structure of the Msx-1 homeodomain/DNA complex. Biochemistry 2001;40:12013-12021.

[15] Hu G, Lee H, Price SM, Shen MM, Abate-Shen C. Msx homeobox genes inhibit differentiation through upregulation of cyclin D1. Development 2001;128:2373-2384.

[16] Hu G, Vastardis H, Bendall AJ, Wang Z, Logan M, Zhang $\mathrm{H}$, et al. Haploinsufficiency of MSX1: a Mechanism for selective tooth agenesis. Mol Cell Biol 1998;18:6044-6051.

[17] Jezewski PA, Vieira AR, Nishimura C, Ludwig B, Johnson $\mathrm{M}$, O'Brien SE, et al. Complete sequencing shows a role for MSX1 in non-syndromic cleft lip and palate. J Med Genet 2003;40(6):399-407.
[18] Jumlongras D, Bei M, Stimson JM, Wang WF, DePalma SR, Seidman CE, et al. A nonsense mutation in MSX1 causes Witkop syndrome. Am J Hum Genet 2001;69:67-74.

[19] Kim HJ, Rice DPC, Kettunen PJ, Thesleff I. FGF-, BMPand Shh-mediated signalling pathways in the regulation of cranial suture morphogenesis and calvarial bone development. Development 1998;125:1241-1251.

[20] Kim JW, Simmer JP, Lin BP, Hu JC. Novel MSX1 frameshift causes autosomal-dominant oligodontia. J Dent Res 2006;85(3):267-271.

[21] Kiyosawa H, Yamanaka I, Osato N, Kondo S, Hayashizaki Y. Antisense transcripts with FANTOM2 clone set and their implications for gene regulation. Genome Res 2003;13:1324-1334.

[22] Kuzuoka M, Takahashi T, Guron C, Raghow R. Murine homeobox-containing gene, Msx-1: analysis of genomic organization, promoter structure, and potential autoregulatory cis-acting elements. Genomics 1994;21:85-91.

[23] Lidral AC, Reising BC. The role of MSX1 in human tooth agenesis. J Dent Res 2002;81:274-278.

[24] Lumsden AG. Spatial organization of the epithelium and the role of neural crest cells in the initiation of the mammalian tooth germ. Development 1988;103 (Suppl): 155-169.

[25] Maas R, Bei M. The genetic control of early tooth development. Crit Rev Oral Biol Med 1997;8:4-39.

[26] MacKenzie A, Ferguson MWJ, Sharpe PT. Hox-7 expression during murine craniofacial development. Development 1991;113:601-611.

[27] MacKenzie A, Leeming GL, Jowett AK, Ferguson MW, Sharpe PT. The homeobox gene Hox 7.1 has specific regional and temporal expression patterns during early murine craniofacial embryogenesis, especially tooth development in vivo and in vitro. Development 1991;111:269-285.

[28] Mina M, Kollar EJ. The induction of odontogenesis in non-dental mesenchyme combined with early murine mandibular arch epithelium. Arch Oral Biol 1987;32:123-127.

[29] Monaghan AP, Davidson DR, Sime C, Graham E, Baldock R, Bhattacharya SS, et al. The Msh-like homeobox genes define domains in the developing vertebrate eye. Development 1991;112:1053-1061.

[30] Mostowska A, Biedziak B, Trzeciak WH. A novel c.581C $>$ T transition localized in a highly conserved homeobox sequence of MSX1: is it responsible for oligodontia? J Appl Genet 2006;47(2):159-164.

[31] Nieminen P, Kotilainen J, Aalto Y, Knuutila S, Pirinen S, Thesleff I. MSXI gene is deleted in Wolf-Hirschhorn Syndrome patients with oligodontia. J Dent Res 2003;82:1013-1017.

[32] Nugent P, Greene RM. MSX-1 gene expression and regulation in embryonic palatal tissue. In Vitro Cell Dev Biol Anim 1998;34:831-835.

[33] Odelberg SJ, Kolhoff A, Keating MT. Dedifferentiation of mammalian myotubes induced by msxl. Cell 2000;103:1099-1109.

[34] Orestes-Cardoso SM, Néfussi JR, Hotton D, Mesbah M, Orestes-Cardoso MDS, Robert B, et al. Postnatal Msxl expression pattern in craniofacial, axial and appendicular 
skeleton of transgenic mice from the first week until the second year. Dev Dyn 2001;221:1-13.

[35] Orestes-Cardoso SM, Néfussi JR, Lézot F, Oboeuf M, Pereira M, Mesbah M, et al. Msxl Is a regulator of bone formation during development and postnatal growth : In vivo investigations in a transgenic mouse model. Conn Tissue Res 2002;43:153-160.

[36] Ramos C, Fernandez-Llebrez P, Bach A, Robert B, Soriano E. Msxl disruption leads to diencephalon defects and hydrocephalus. Dev Dyn 2004;230:446-460.

[37] Ramos C, Martinez A, Robert B, Soriano E. Msxl expression in the adult mouse brain: characterization of populations of beta-galactosidase-positive cells in the hippocampus and fimbria. Neuroscience 2004;127: 893-900.

[38] Reginelli AD, Wang YQ, Sassoon D, Muneoka K. Digit tip regeneration correlates with regions of Msxl (Hox 7) expression in fetal and newborn mice. Development 1995;121:1065-1076.

[39] Robert B, Sassoon D, Jacq B, Gehring W, Buckingham M. Hox-7, a mouse homeobox gene with a novel pattern of expression during embryogenesis. Embo J 1989;8:91-100

[40] Satokata I, Maas R. Msxl deficient mice exhibit cleft palate and abnormalities of craniofacial and tooth development. Nat Genet 1994;6:348-356.

[41] Sharpe PT. Homeobox genes and orofacial development. Connect Tissue Res 1995;32:17-25.

[42] Shashikant CS, Utset MF, Violette SM, Wise TL, Einat P, Einat M, et al. Homeobox genes in mouse development. Crit Rev Eukaryot Gene Expr 1991;1:207-245.

[43] Shetty S, Takahashi T, Matsui H, Ayengar R, Raghow $\mathrm{R}$. Transcriptional autorepression of Msxl gene is mediated by interactions of Msxl protein with a multi-protein transcriptional complex containing TATA-binding protein, Spl and cAMP-response-element-binding proteinbinding protein (CBP/p300). Biochem J 1999;339 (Pt 3): 751-758.

[44] Thesleff I, Mikkola M. The role of growth factors in tooth development. Int Rev Cytol 2002;217:93-135.

[45] Thesleff I, Vaahtokari A, Kettunen P, Aberg T. Epithelialmesenchymal signalling during tooth development. Connect Tissue Res 1995;32:9-15.

[46] Thomas BL, Porteus MH, Rubenstein JLR, Sharpe PT. The spatial localization of Dlx2 during tooth development. Connect Tissue Res 1995;32:27-34.

[47] Thomas BL, Tucker AS, Ferguson C, Qiu M, Rubenstein JLR, Sharpe PT. Molecular control of odontogenic patterning: Positional dependent initiation and morphogenesis. Eur J Oral Sci 1998;106 (Suppl 1):44-47.

[48] Tongkobpetch S, Siriwan P, Shotelersuk V. MSX1 mutations contribute to nonsyndromic cleft lip in a Thai population. Hum Genet 2006;51(8):671-676.

[49] Tribulo C, Aybar MJ, Sanchez SS, Mayor R. A balance between the anti-apoptotic activity of Slug and the apoptotic activity of msxl is required for the proper development of the neural crest. Dev Biol 2004;275:325-342.

[50] Tucker A, Sharpe PT. The cutting-edge of mammalian development; how the embryo makes teeth. Nat Rev Genet 2004;5:499-508.

[51] Tureckova J, Sahlberg C, Aberg T, Ruch JV, Thesleff I, Peterkova R. Comparison of expression of the msx-1, msx-2, BMP-2 and BMP-4 genes in the mouse upper diastemal and molar tooth primordia. Int J Dev Biol 1995;39:459-468.

[52] Vainio S, Karavanova I, Jowett A, Thesleff I. Identification of BMP4 as a signal mediating secondary induction between epithelial and mesenchymal tissues during early tooth development. Cell 1993;75:45-58.

[53] Van den Boogaard MJ, Dorland M, Beermer FA, van Amstel HK. MSXl mutation is associated with orofacial clefting and tooth agenesis in humans. Nat Genet 2000;24(4):342-343

[54] Vastardis H, Karimbux N, Guthua SW, Seidman JG, Seidman CE. A human MSX1 homeodomain missense mutation causes selective tooth agenesis. Nat Genet 1996;13:417-421.

[55] Vi-Fane B. Étude des transcrits sens et antisens de l'homéogène Msxl dans le tissu osseux oro-facial. Mémoire de DEA de Biologie Orale et Ostéo-articulaire, Biomatériaux et Biofonctionnalité. Paris: Univ Denis Diderot, 2001.

[56] Vi-Fane B, Néfussi JR, Orestes-Cardoso S, Hotton D, Robert B, Chabre $\mathrm{C}$, et al. Pour une approche génétique et expérimentale de la croissance squelettique cranio-faciale : étude du rôle de l'homéogène divergent Msx1. Orthod Fr 2003;74:103-113.

[57] Wang W, Chen X, Xu H, Lufkin T. Msx3: a novel murine homologue of the Drosophila msh homeobox gene restricted to the dorsal embryonic central nervous system. Mech Dev 1996;58:203-215.

[58] Weiss KM, Bollekens J, Ruddle F, Takashita K. Distalless and other homeobox genes in the development of the dentition. J Experimental Zoology 1994;270: 273-284

[59] Weiss KM, Stock DW, Zhao Z. Dynamic interactions and the evolutionary genetics of dental patterning. Crit Rev Oral Biol Med 1998;9(4):369-398.

[60] Werner A, Berdal A. Natural antisense transcripts: sound or silence? Physiol Genomic 2005;23(2): 125-131.

[61] Zhang H, Catron KM, Abate-Shen C. A role for the Msx1 homeodomain in transcriptional regulation: residues in the N-terminal arm mediate TATA binding protein interaction and transcriptional repression. Proc Natl Acad Sci U S A 1996;93:1764-1769.

[62] Zhang Z, Song Y, Zhao X, Zhang X, Fermin C, Chen Y. Rescue of cleft palate in Msxl-deficient mice by transgenic Bmp4 reveals a network of BMP and Shh signaling in the regulation of mammalian palatogenesis. Development 2002;129:4135-4146. 